\title{
Cone beam CT with zonal filters for simultaneous dose reduction, improved target contrast and automated setup in radiotherapy.
}

\author{
C J Moore, T E Marchant and A M Amer. \\ North Western Medical Physics, Christie Hospital NHS Trust, Manchester, UK.
}

\begin{abstract}
.
Cone beam CT (CBCT) using a zonal filter is introduced. The aims are reduced concomitant imaging dose to the patient, simultaneous control of body scatter for improved image quality in the tumour target zone and preserved set-up detail for radiotherapy. Aluminium transmission diaphragms added to the CBCT $\mathrm{X}$-ray tube of the Elekta Synergy ${ }^{\mathrm{TM}}$ linear accelerator produced an un-attenuated beam for a central 'target zone' and a partially-attenuated beam for an outer 'setup zone'. Imaging doses and contrast noise ratios (CNR) were measured in a test phantom for transmission diaphragms 12 and $24 \mathrm{~mm}$ thick, for 5 and $10 \mathrm{~cm}$ long target zones. The effect on automatic registration of zonal CBCT to conventional CT was assessed relative to full field and lead collimated images of an anthropomorphic phantom. Doses along the axis of rotation were reduced by up to $50 \%$ in both target and setup zones, and weighted dose (two thirds surface dose plus one third central dose) was reduced by $10-20 \%$ for a $10 \mathrm{~cm}$ long target zone. CNR increased by up to $15 \%$ in zonally filtered CBCT images compared to full field images. Automatic image registration remained as robust as that with full field images and was superior to CBCT coned down using leadcollimation. Zonal CBCT significantly reduces imaging dose and is expected to benefit radiotherapy through improved target contrast, required to assess target coverage, and wide-field edge detail, needed for robust automatic measurement of patient set-up error.
\end{abstract}

Keywords: cone-beam CT, radiotherapy set-up

This is an author-created, un-copyedited version of an article accepted for publication in Physics in Medicine and Biology. IOP Publishing Ltd is not responsible for any errors or omissions in this version of the manuscript or any version derived from it. The definitive publisher-authenticated version is available online at http://dx.doi.org/10.1088/0031-9155/51/9/005. 


\section{$1 \quad$ Introduction}

A recent advance in radiotherapy imaging has been the integration of kilovoltage $\mathrm{x}$-ray sources and amorphous silicon imaging panels onto commercially available medical linear accelerator gantries. This allows digital, rotational fluoroscopy to be performed in support of cone-beam CT (CBCT) reconstructive imaging (Jaffray et al., 2002). Since CBCT images are three dimensional (3D) they can be compared to treatment planning images to determine patient set-up errors from the displacement of bony anatomy in all directions (Marchant et al., 2004). Alternatively they can be used to assess the position of a target structure using soft tissue detail that is much improved compared to the mega-voltage imaging modalities (Smitsmans et al., 2004). It has been shown that sufficient bony edge detail can be obtained for patient setup using a very low dose CBCT scanning protocol (Sykes et al., 2005). However, the imaging of soft tissue target organs, adjacent to others with similar attenuation characteristics, such as the prostate, requires a higher dose CBCT scan (Amer et al., 2005). This is a site that is already challenging for conventional crosssectional CT let alone CBCT.

A timely reminder of the need for image guided radiotherapy (IGRT) has been forcefully demonstrated by the verification of Intensity Modulated Radiotherapy prostate treatment failure due to rectal changes during therapy (de Crevoisier et al., 2005, Roach, 2005). Several authors have discussed the use of image-guidance before each treatment fraction to allow on-line correction of patient position (Oldham et al., 2005, de Crevoisier et al., 2005, Ghilezan et al., 2004). However, the repeated use of $x$-ray imaging during a course of treatment has also raised concerns over the concomitant imaging dose received by the patient (Aird, 2004). In the UK, as in Europe, there is a statutory responsibility to keep such doses as low as reasonably practical (EU, 1997, HMSO, 2000), both in diagnostic radiology and radiotherapy.

CBCT images are reconstructed from a sequence of wide field projection images, and thus image quality is degraded by scattered radiation to a greater degree than in conventional CT, where imaging uses a narrowly collimated fan-beam (Endo et al., 2001, Siewerdsen and Jaffray, 2001). Scattered radiation can be reduced by applying the radiological practice of coning down to smaller fields of view (Siewerdsen and Jaffray, 2001), however this reduces the amount of information available for image registration (c.f. treatment beam portal vs. wide field portal images). Anti-scatter grids have also been applied in CBCT (Siewerdsen et al., 2004), and found to be of limited use due to the trade-off between increased contrast and increased noise. Dual exposure CBCT, the 3D analog of dual exposure electronic portal imaging, has been reported (Letourneau et al., 2005), where a reduced field of view image of the target is superimposed into a lower dose, wide field image. This technique combines high quality imaging of the target with wide field detail for required for set-up measurement, but the use of two CBCT images inevitably increases patient imaging dose still further.

Here the concept of zonal cone beam tomography is introduced, using partial transmission filters or diaphragms to reduce the exposure to the periphery of the imaging field, thus removing scattered radiation at source and reducing dose. The aim is to retain the wide field bony detail necessary for patient set-up, and to simultaneously improve target zone contrast due to the reduction in scattered radiation. This also reduces dose to the bulk of the patient, allowing more frequent use of CBCT. 


\section{$2 \quad$ Materials and Methods}

\subsection{Zonal filter design}

The zonal filter consists of two aluminium transmission diaphragms that can be fixed to the $\mathrm{kV} \mathrm{x}$ ray tube as an external beam collimation device, as shown in Figure 1. The filters are aligned so as to restrict the central field of view in the longitudinal direction, see Figure 2. This creates a 'target zone' with an unattenuated imaging beam and a partially attenuated beam for an outer 'setup zone'. The spacing of the aluminium filters can be adjusted to customize the length of the target zone. Two thicknesses, $12 \mathrm{~mm}$ and $24 \mathrm{~mm}$, of aluminium have been tested in this initial study. This corresponds to approximately 2 and 4 Half Value Layers (HVL) for the $120 \mathrm{kV}$ x-ray beam used here. The aluminium filters have a bevelled inner edge, as shown in Figure 1c, to reduce the discontinuity between differentially irradiated zones in the $\mathrm{x}$-ray projection images acquired for $\mathrm{CBCT}$ reconstruction.

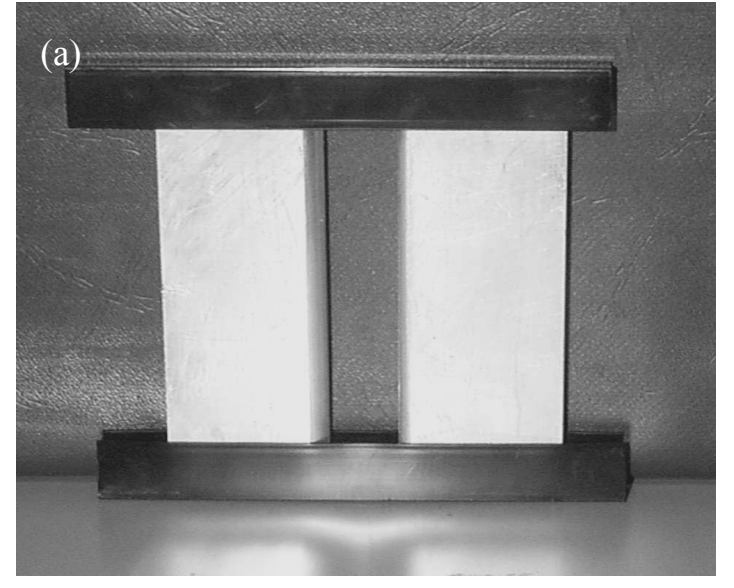

(c)

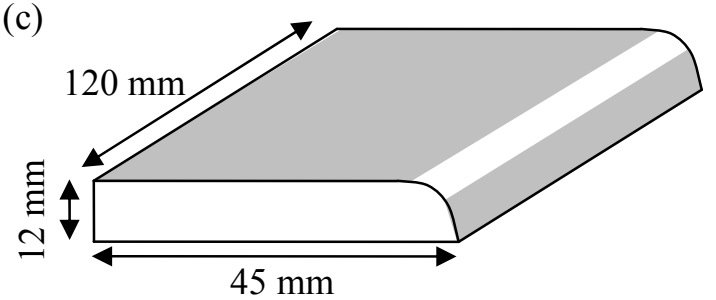

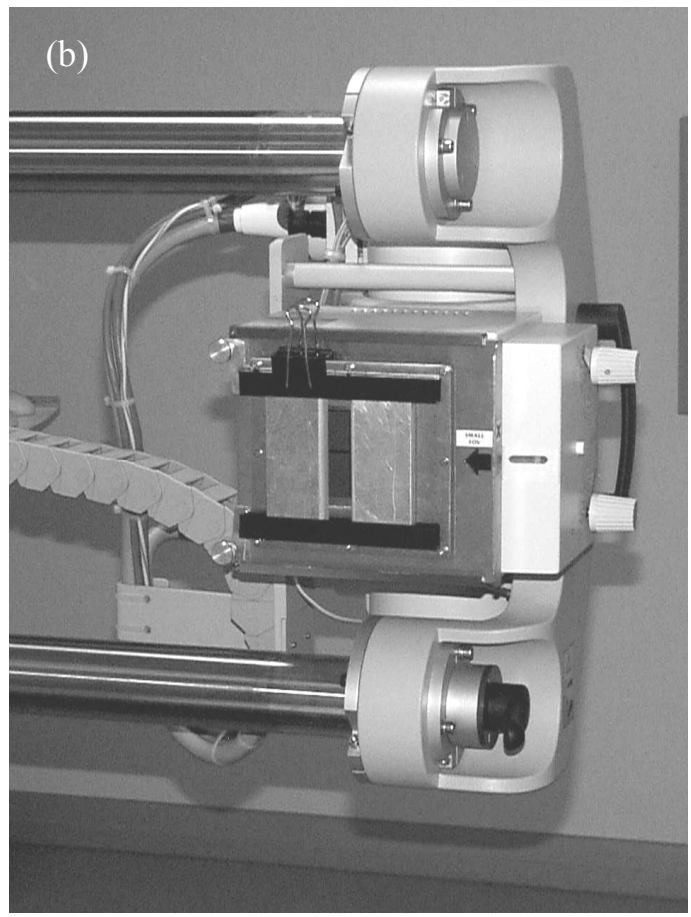

Figure 1: (a) Aluminium filters with adjustable spacing. (b) The filters attached to the $\mathrm{kV} x$-ray tube (c) shaping of filter edges. 


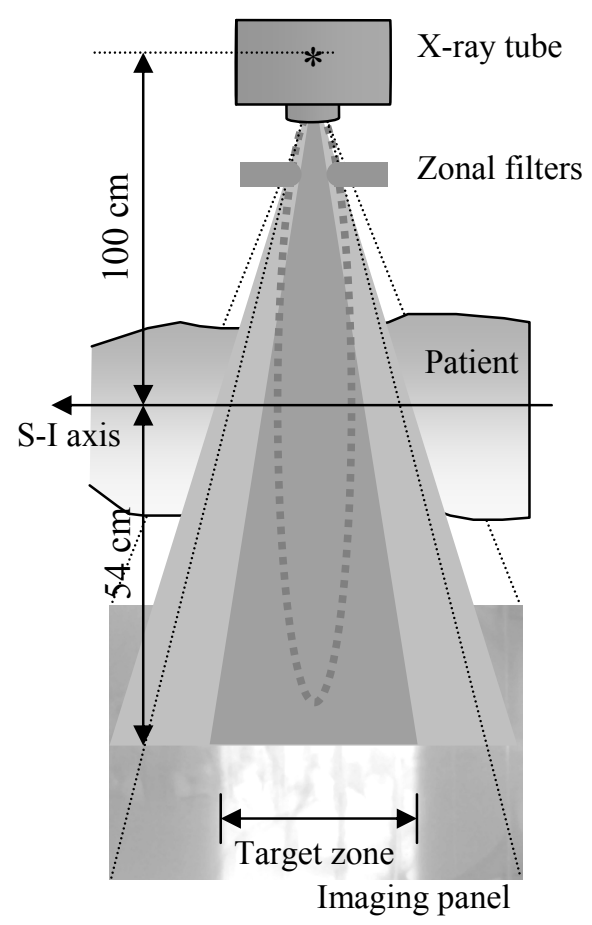

Figure 2: Schematic representation of how zonal filters collimate $x$-ray imaging beam into unattenuated target zone and partially attenuated setup zones (not to scale).

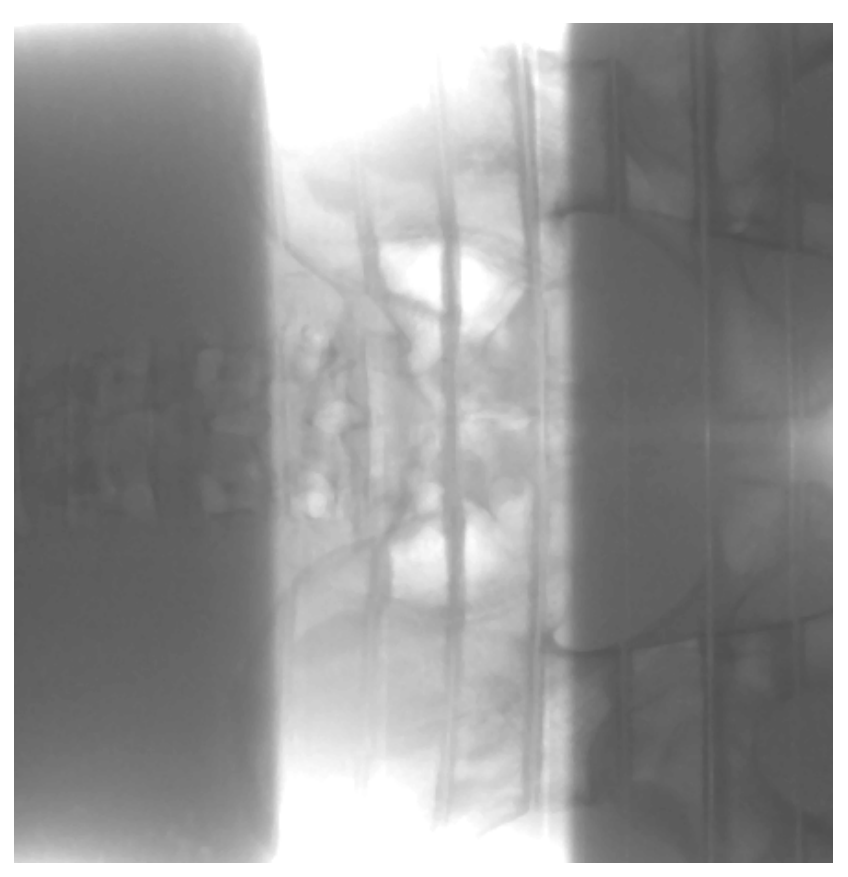

Figure 3: Projection image of Rando phantom using zonal filter.

\subsection{Image acquisition and reconstruction}

CBCT images were acquired with the Elekta Synergy ${ }^{\mathrm{TM}}$ imaging system at Christie Hospital. Xray projection images were acquired using the system's amorphous silicon panel imager set to 
provide $512 \times 512$ resolution, with equivalent pixel size $0.8 \times 0.8 \mathrm{~mm}$ at the imaging panel. A typical projection image using the zonal filter of the Rando anthropomorphic phantom is shown in Figure 3. The length of the central zone is $10 \mathrm{~cm}$ at the isocentre. The signal level in the outer setup zones is observed to be lower than in the central zone, but some detail of the bony anatomy is still visible. The Elekta Synergy has its $\mathrm{kV} \mathrm{X}$-ray tube mounted with the anode-cathode axis tangential to the circular scanning trajectory. This means that the dose effects due to the anode heel effect average out for a circular scan. Volume images were reconstructed using Feldkamp filtered back projection (Feldkamp et al., 1984). This yielded a CBCT of 256x256x256 voxels cubed, with voxel size $1 \mathrm{~mm}$ is all directions. Thus, the full length of the FOV is $25.6 \mathrm{~cm}$. A simple de-dishing algorithm was applied to the reconstructed slice images to reduce the dishing artefact caused by scatter, beam-hardening and local tomography in the CBCT images. This artefact has a circular symmetry about the centre of each slice image and, uncorrected, would cause the voxel values to be elevated towards the edge of the image. The artefact was corrected by scaling the voxel values in each transverse slice using a radially symmetric quadratic function centred on the axis of rotation. The magnitude of the correction was selected interactively by the user. The dish artefact is distinct from ring artefacts caused by imperfections in detector calibration, which take the form of circular structures at a fixed radius.

\subsection{Target zone contrast to noise ratio}

A phantom was designed and constructed in-house to allow the measurement of contrast and noise in the reconstructed images. The phantom was intended to mimic pelvic anatomy in a large patient, a situation where it is difficult to obtain good contrast using current techniques. The phantom is illustrated in Figure 4. It is constructed from Perspex (Plexiglass), with various synthetic material inserts to provide contrast differences in the reconstructed images. The phantom also contains two high density plastic (Teflon) inserts to mimic the femoral heads, which impact image quality at the centre of the pelvis. The phantom is $20 \mathrm{~cm}$ in length, and all images were acquired with additional Perspex placed at either end of the phantom. This extends the length of the phantom to greater than the maximum CBCT field length and provides a realistic scatter field.
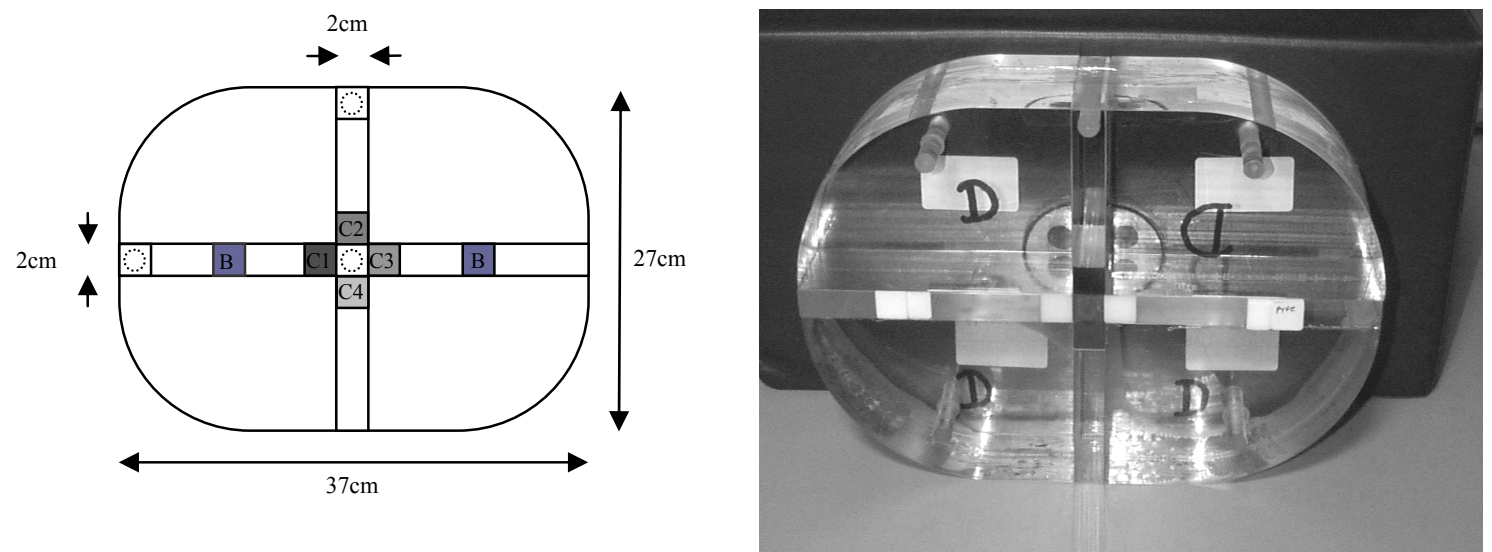

Figure 4: Perspex phantom used to measure contrast in reconstructed cone-beam CT images.

Images were acquired of the phantom for target zone lengths of $5 \mathrm{~cm}$ and $10 \mathrm{~cm}$. At each field size, images were acquired with $12 \mathrm{~mm}$ and $24 \mathrm{~mm}$ thick aluminium filters. Images were also acquired with the X-ray beam fully collimated using lead diaphragms to the same size as the target zones in the zonal filter images. This gives the maximum reduction in scatter from outside the target zone, and hence the maximum increase in target zone image quality, in the situation 
where no image information is retained from the outside of the target zone. The centre of the phantom was aligned with the isocentre, so that each of the contrast inserts were at the same radius from the centre of the image.

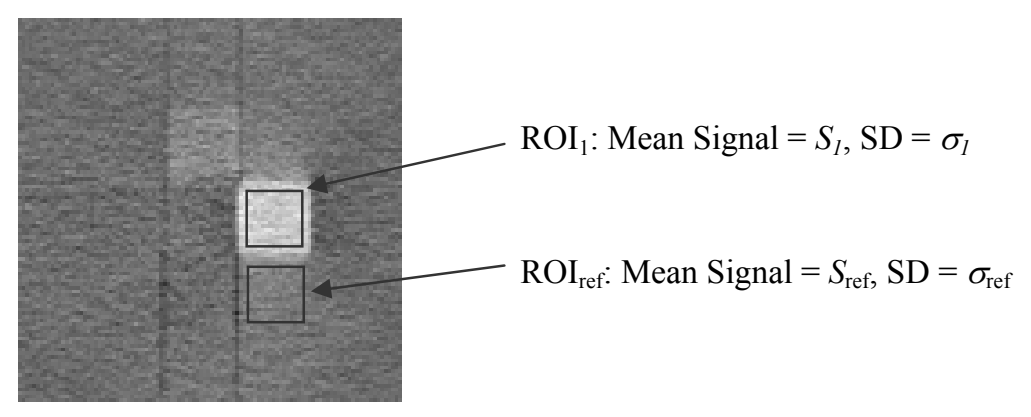

Figure 5: Slice image of central region of contrast phantom, showing definition of regions used to calculate contrast to noise ratio.

For each image the average, $S_{i}$, and standard deviation, $\sigma_{i}$, of the voxel values in contrast insert $i$ were found, see Figure 5. A reference region was also defined in the Perspex bulk of the phantom.

The contrast of each region, $C_{i}$, is defined as the difference between the average voxel value in that region and the average value in the reference region,

$$
C_{i}=S_{i}-S_{\text {ref. }}
$$

The contrast to noise ratio, $C N R_{i}$, is defined as the ratio of that region's contrast to the standard deviation of the voxel values in the reference region,

$$
C N R_{i}=\frac{C_{i}}{\sigma_{\text {ref }}}
$$

CNR is the most important measure of image quality for these CBCT images because it relates to the visibility of relatively large (compared to the spatial resolution) low contrast objects. Absolute contrast is not a good measure of this because the voxel values are not calibrated and can vary from image to image due to normalisation differences during reconstruction.

For each zonal filter configuration (target zone length, and filter thickness), a contrast improvement factor (CIF), CIF , is defined as the percentage increase in CNR compared to the full FOV with no zonal filter in place,

$$
C I F_{i}=\frac{C N R_{i}-C N R_{\text {Full }}}{C N R_{\text {Full }}} \times 100 \%
$$

where $C N R_{\text {Full }}$ is the CNR achieved with the full FOV and no zonal filter in place.

\subsection{Spatial resolution}

Spatial resolution of the reconstructed zonal filter images was assessed using a Catphan CT phantom resolution module (CTP528, The Phantom Laboratory, Salem, NY). This was done using CBCT images reconstructed with $0.5 \mathrm{~mm}$ voxel size. Resolution was assessed both in the 
target and setup zones for $12 \mathrm{~mm}$ and $24 \mathrm{~mm}$ thick zonal filters with a target zone length of $5 \mathrm{~cm}$, and for the uncollimated field. The maximum number of line pairs per $\mathrm{cm}$ visible was assessed visually for each image, and f50, the frequency at which the modulation transfer function falls to $50 \%$, was also measured.

\subsection{Imaging dose}

The ion chamber used (3CT with 9010 dosimeter, Radcal, USA) has a sensitive length of approximately $10 \mathrm{~cm}$. In order to measure target zone doses for field sizes less than $10 \mathrm{~cm}$, a lead shield was designed with a $1 \mathrm{~cm}$ gap, as shown in Figure 6. The lead shield has a thickness of 2 $\mathrm{mm}$, which attenuates $150 \mathrm{keV}$ x-rays to $1 \%$ of the original intensity, and even less than this for lower energy x-rays. Hence there should be very little penetration through the shield. Only xrays entering through the gap in the shield will cause a dose to be measured. The shielded chamber was only used to measure relative doses, hence it was not necessary to calibrate it with the shield in place.
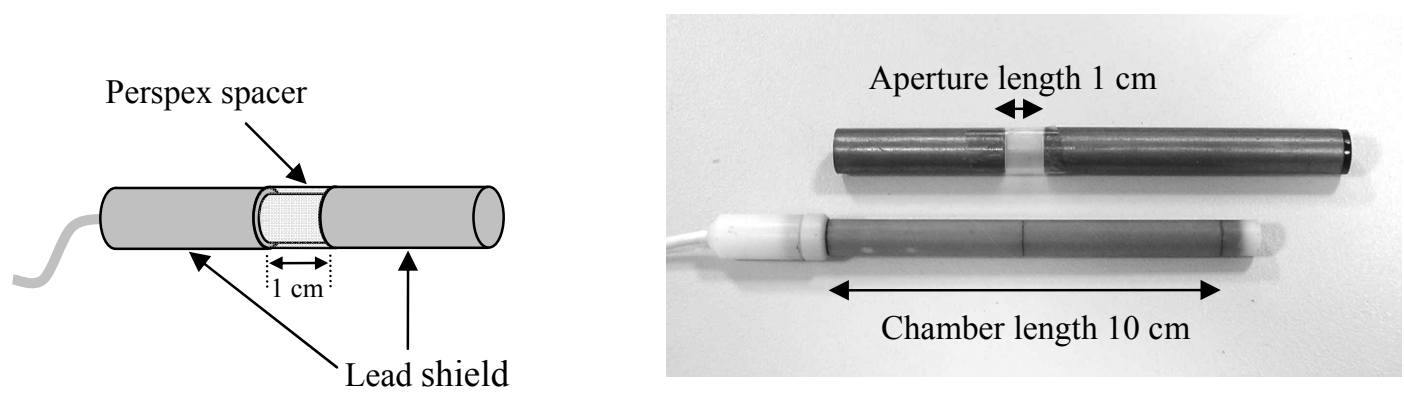

Figure 6: $\mathrm{kV}$ ion chamber with $1 \mathrm{~cm}$ opening lead shield.

Imaging dose measurements were made using two different phantoms. The first, a $32 \mathrm{~cm}$ diameter cylindrical CT Dose Index (CTDI) phantom made of Perspex containing chamber cavities at the centre and at peripheral positions $1 \mathrm{~cm}$ below the surface (IEC, 1994), is shown in Figure $7 \mathrm{a}$. The shielded ion chamber was used to measure the central and peripheral dose within the target zone (position A in Figure $7 \mathrm{~b}$ ). The weighted dose was also calculated (2/3 peripheral dose $+1 / 3$ central dose) according to the recommendations contained in (EU, 1999). Dose measurements were also made using the contrast phantom described in section 2.3 , which also contains cylindrical holes into which an ion chamber can be placed. The ion chamber can be placed at the centre of the phantom or $1 \mathrm{~cm}$ deep at the lateral and anterior surfaces. The ion chamber holes were plugged with Perspex rods when not in use. Dose measurements with this phantom were made within the target zone and also with the chamber offset so that the sensitive volume is situated underneath the transmission filters, within the setup zone (position B in Figure $7 b$ ). Additional Perspex scatter material was placed either side (longitudinally) of both phantoms to provide scatter from the edges of the FOV, as would be the case in pelvic imaging. It is important to ensure that the scatter material extends beyond the edge of x-ray beam, to avoid underestimation of the scattered radiation (Mori et al., 2005). Measurements were made for 12 and $24 \mathrm{~mm}$ thickness aluminium zonal filters and for lead collimated imaging fields using the $\mathrm{kV}$ tube's internal lead collimators. Doses were measured for target zone lengths of $5 \mathrm{~cm}$ and $10 \mathrm{~cm}$ and for the full field of $25.6 \mathrm{~cm}$. 
(a)

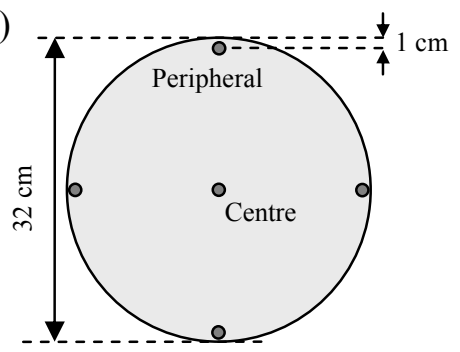

(b)

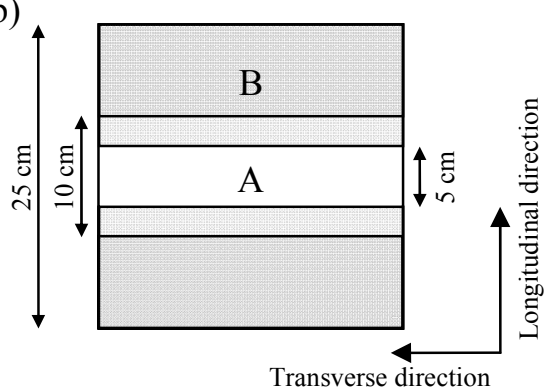

Figure 7: (a) Axial view of CTDI phantom used for dose measurements. (b) Beams eye view of imaging FOV showing areas shielded by zonal filter, and positions of relative dose measurements made using shielded $\mathrm{kV}$ ion chamber.

\subsection{Image registration}

Images of the Alderson Rando anthropomorphic phantom pelvis were used to test the effect of the zonal filter on automatic image registration of CBCT images. CBCT images were acquired using the full $25.6 \mathrm{~cm}$ FOV, and using the zonal filter with $12 \mathrm{~mm}$ and $24 \mathrm{~mm}$ thick aluminium filters, and with $5 \mathrm{~cm}$ and $10 \mathrm{~cm}$ central zone lengths. Lead collimated images with FOV length $5 \mathrm{~cm}$ and $10 \mathrm{~cm}$ were also acquired. The Rando phantom was not moved between acquisitions of these images. All of the images were reconstructed and imported into the Philips Pinnacle ${ }^{\mathrm{TM}}$ treatment planning system. A conventional CT scan of the Rando phantom was also acquired with $5 \mathrm{~mm}$ thick slices at $5 \mathrm{~mm}$ intervals. This was also imported into Pinnacle. The Philips Syntegra ${ }^{\mathrm{TM}}$ image registration tools were used to register each of the CBCT scans to the conventional CT scan using the Local Correlation algorithm (Netsch et al., 2001, Rosch et al., 2003). The registration of the full field CBCT scan to the conventional CT was assessed visually to be a good match of bony anatomy. This was taken to be the "correct" registered position, and the same transformation was applied to each of the other CBCT scans. Since the phantom was not moved between CBCT scans, this position was a good match for all scans. The robustness of the registration was tested by adding random initial offsets taken from a normal distribution to the CBCT scans, to test how often successful automatic registration could be achieved. Successful registration was defined as being within $1 \mathrm{~mm}$ of the "correct" position in each direction. This grading of the automatic registrations as either success or failure was justified because the failures were very obvious. "Near misses" were very rare. Random initial offsets were taken from normal distributions with standard deviations $2 \mathrm{~mm}, 5 \mathrm{~mm}, 10 \mathrm{~mm}$, and $20 \mathrm{~mm}$. Ten samples were taken for each value of standard deviation.

\section{$3 \quad$ Results}

\subsection{Target zone contrast to noise ratio}

Figure 8 shows the contrast improvement factor for the different zonal filter configurations that were tested. The error bars in Figure 8 represent one standard error about the mean of the distribution of CIF factors measured from 20 image slices through the contrast inserts. It can be seen that significant improvements in CNR of up to $15 \%$ are achieved by using the zonal filter. The lead collimated $5 \mathrm{~cm}$ field shows that a CNR improvement of around $30 \%$ can be achieved with non-transmission filters. The important difference is that the zonal filter images retain sufficient edge detail for bony anatomy matching throughout the whole $25 \mathrm{~cm}$ field length. The lead collimated image retains no image information outside the $5 \mathrm{~cm}$ target zone. CNR measurements were also made with the phantom offset longitudinally so that the contrast inserts were located within the setup zone (i.e. underneath the aluminium filters). The reduction in CNR was measured to be $37 \%$ and $60 \%$ for $12 \mathrm{~mm}$ and $24 \mathrm{~mm}$ thick filters respectively. 


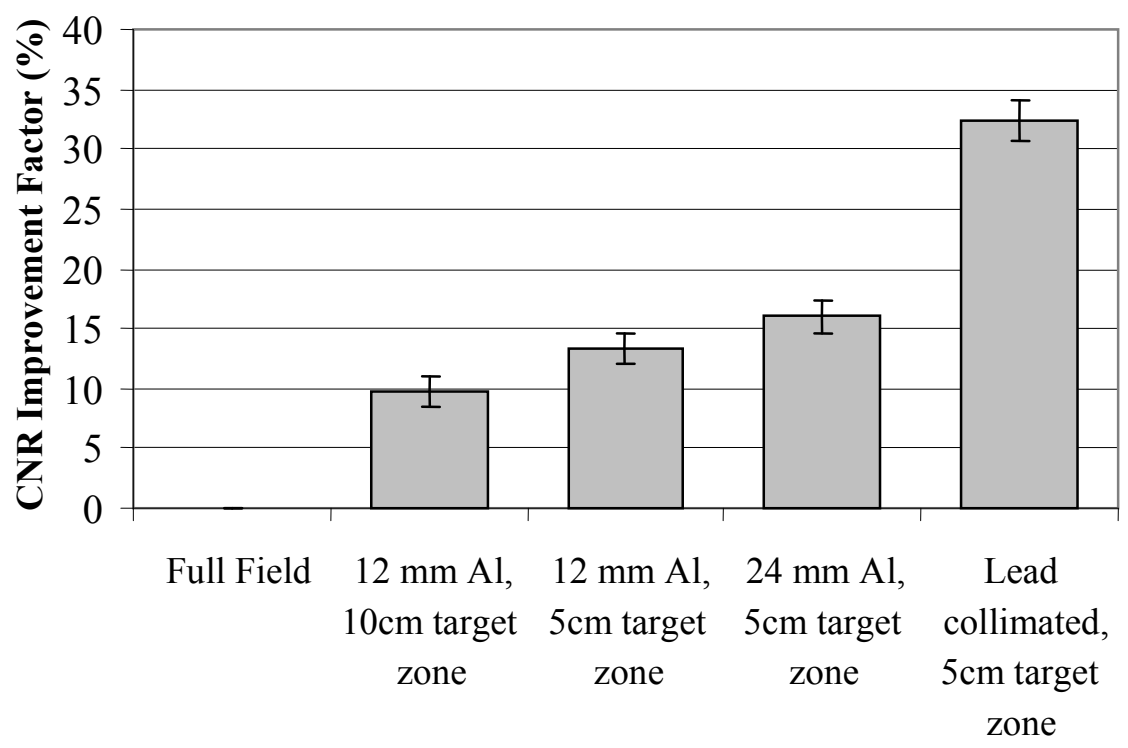

Figure 8: Contrast improvement factor relative to full field image for various different zonal filter configurations. The error bars indicate one standard error about the mean.

\subsection{Spatial resolution}

Figure 9 shows images of the resolution test objects with spatial frequency 4 to 10 line pairs per $\mathrm{cm}$ for CBCT images (a) at the central slice with no zonal filter (b) at the central slice with 12 $\mathrm{mm}$ thick zonal filter with $5 \mathrm{~cm}$ target zone length (c) at the central slice with $24 \mathrm{~mm}$ thick zonal filter with $5 \mathrm{~cm}$ target zone length (d) in the setup-zone with no filter (e) in the setup-zone with $12 \mathrm{~mm}$ thick zonal filter with $5 \mathrm{~cm}$ target zone length and (f) in the setup zone with $24 \mathrm{~mm}$ thick zonal filter with $5 \mathrm{~cm}$ target zone length. For the setup zone images the resolution test object was approximately $9 \mathrm{~cm}$ from the central slice. Visual inspection shows that the maximum frequency visible is $8 \mathrm{lp} / \mathrm{cm}$ in all cases. The f50 value of around $4 \mathrm{lp} / \mathrm{cm}$ is maintained even under the 24 $\mathrm{mm}$ zonal filter, although the images are observed to be more noisy in this case. 


\begin{tabular}{|c|c|c|c|c|c|c|}
\hline $\begin{array}{c}\text { Freq } \\
{[1 \mathrm{p} / \mathrm{cm}]}\end{array}$ & $\begin{array}{c}\text { (a) } \\
\text { No Filter } \\
\text { Target } \\
\text { zone }\end{array}$ & $\begin{array}{c}\text { (b) } \\
12 \mathrm{~mm} \mathrm{~A} \\
5 \mathrm{~cm} \\
\text { Target } \\
\text { zone }\end{array}$ & \begin{tabular}{|c|}
$(\mathrm{c})$ \\
$24 \mathrm{~mm} \mathrm{Al}$ \\
$5 \mathrm{~cm}$ \\
Target \\
zone \\
\end{tabular} & $\begin{array}{c}(\mathrm{d}) \\
\text { No Filter } \\
\text { Setup } \\
\text { zone }\end{array}$ & $\begin{array}{c}(\mathrm{e}) \\
12 \mathrm{~mm} \mathrm{Al} \\
5 \mathrm{~cm} \\
\text { Setup } \\
\text { zone }\end{array}$ & $\begin{array}{c}(\mathrm{f}) \\
24 \mathrm{mmAl} \\
5 \mathrm{~cm} \\
\text { Setup } \\
\text { zone }\end{array}$ \\
\hline 4 & & & & & & \\
\hline 5 & & & & & & \\
\hline 6 & & & & & & \\
\hline 7 & & & & & & \\
\hline 8 & & & & & & \\
\hline 9 & & & & & & \\
\hline 10 & & & & & & \\
\hline $\begin{array}{l}\text { Max } \\
\mathrm{lp} / \mathrm{cm}\end{array}$ & 8 & 8 & 8 & 8 & 8 & 8 \\
\hline $\begin{array}{c}\mathrm{f} 50 \\
{[1 \mathrm{p} / \mathrm{cm}]}\end{array}$ & 4.2 & 4.3 & 3.9 & 4.2 & 4.3 & 3.9 \\
\hline
\end{tabular}

Figure 9: Catphan spatial resolution test objects imaged with no zonal filter (a \& d), with $12 \mathrm{~mm}$ thick zonal filter (b \& e) and with $24 \mathrm{~mm}$ thick zonal filter (c \& f). Images (a) to (c) are with the test object aligned with the central slice (i.e. within the target zone), images (d) to (f) are with the test object offset $9 \mathrm{~cm}$ in the longitudinal direction (i.e. within the setup zone). All zonal filter images used a central field length of $5 \mathrm{~cm}$.

\subsection{Imaging dose}

Figure 10 shows the dose relative to the full field for different zonal filter configurations (target zone length and filter thickness) measured in the $32 \mathrm{~cm}$ diameter CTDI phantom. Results are shown for chamber positions at the centre of the phantom and also at the peripheral position (1 $\mathrm{cm}$ below the surface). Dose at the centre of the phantom is reduced by approximately $50 \%$ for the $24 \mathrm{~mm}$ thick zonal filter with a $5 \mathrm{~cm}$ central field length. This decrease in dose is entirely due to the reduction of scattered radiation from the edges of the field. For the case of a lead collimated $5 \mathrm{~cm}$ field the central dose is reduced by approximately $70 \%$. The variation of dose 
with target zone length changes faster at small lengths that at larger lengths due to the exponential attenuation of scattered radiation reaching the detector.

The reduction in dose at the peripheral position ( $1 \mathrm{~cm}$ below the surface of the phantom) is roughly half of that achieved at the central position. This is because a greater proportion of the dose is from primary radiation close to the surface, so the reduction in scattered radiation is less significant.

Figure 10c shows the weighted doses resulting from the various zonal filter configurations. The zonal filter gives weighted dose reduction between $10 \%$ and $30 \%$ depending on the filter thickness and field length. The lead collimated field (no image information retained outside central field) gives weighted dose reduction of up to $40 \%$.

(a) Central doses

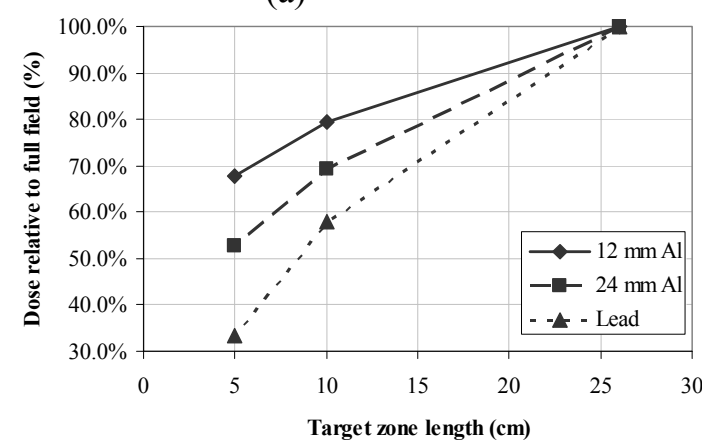

(c) Weighted doses

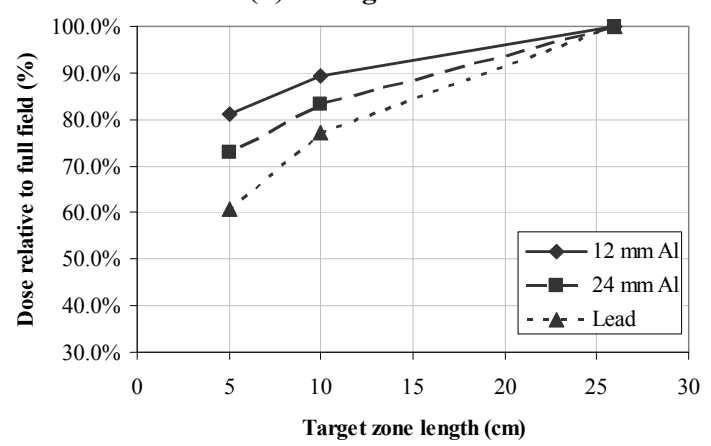

(b) Peripheral doses

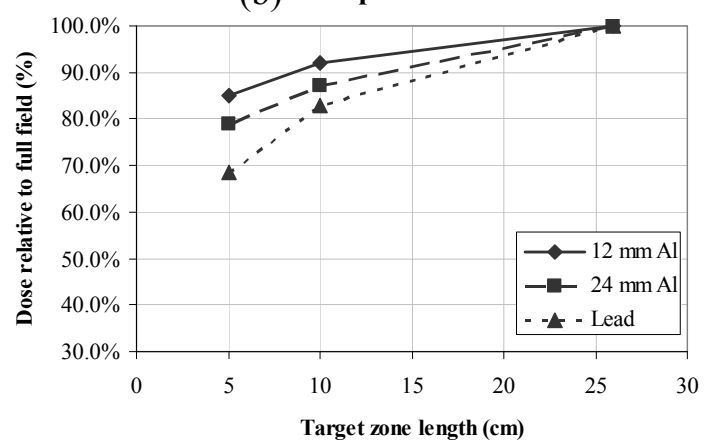

Figure 10: Dose measured in $32 \mathrm{~cm}$ diameter CTDI phantom as a function of target zone field length relative to full field for $12 \mathrm{~mm}$ and $24 \mathrm{~mm}$ thickness zonal filters, and lead collimators (a) at centre of phantom (b) with chamber at peripheral position in phantom (c) weighted dose (combined central and peripheral).

Figure 11 shows the doses measured at the centre of the Perspex contrast phantom for different filter thicknesses and target zone length. It can be seen that significant reductions in central dose of approximately $50 \%$ are achieved both in the target zone and in the setup zone of the field. The large dose reduction in the setup zone is expected due to the shielding of primary radiation, with the remaining dose contributed from photons penetrating the transmission filter (approximately $25 \%$ of the incident radiation for the $12 \mathrm{~mm}$ thick filter), and scattered radiation from other parts of the field. The difference in primary radiation penetrating the filters accounts for the larger difference in dose between the two filter thicknesses for this chamber position. The dose in the centre of the field is also greatly reduced. Here the primary radiation has not been attenuated, and the reduction in dose must be explained by the reduction in scatter dose from the 
edges of the field. The difference between the two filter thicknesses is much smaller here. The large reduction in central dose caused purely by cutting scatter is an illustration of the significant effect of scattered radiation in CBCT. The reduction in central dose using zonal filters was greater when measured using the contrast phantom. This is probably because this phantom is larger than the CTDI phantom, and thus provides more scatter from the edges of the FOV.

(a) Target zone

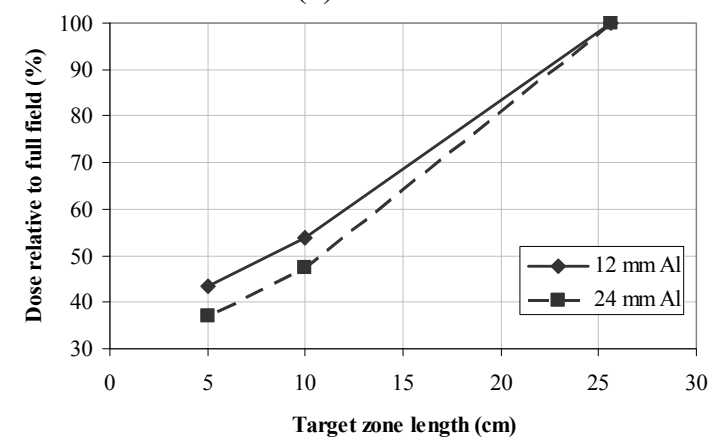

(b) Setup zone

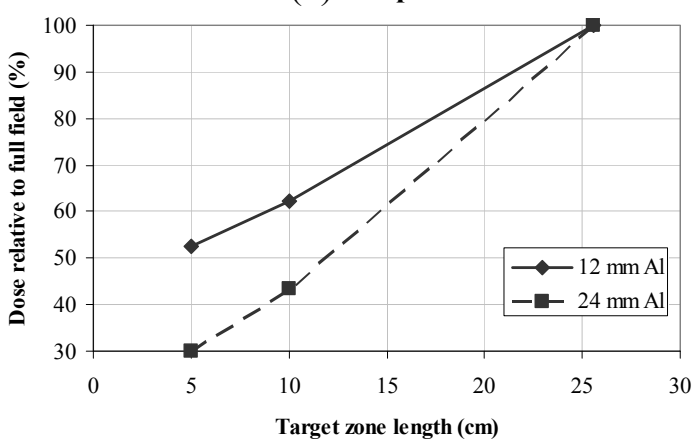

Figure 11: Doses measured at centre of Perspex contrast phantom as a function of target zone field length relative to full field for $12 \mathrm{~mm}$ and $24 \mathrm{~mm}$ thickness zonal filters (a) at centre of field (b) in the setup zone (under transmission filters).

\subsection{Image registration}

Figure 12 shows sagittal $1 \mathrm{~mm}$ slices through CBCT image volumes of the Rando phantom using zonal filters of thickness $12 \mathrm{~mm}$ and $24 \mathrm{~mm}$. The superior and inferior zones where primary radiation is partially shielded by the filter can be identified by the increased noise. However, the edge detail necessary to identify bony anatomy is still clearly visible in these areas.

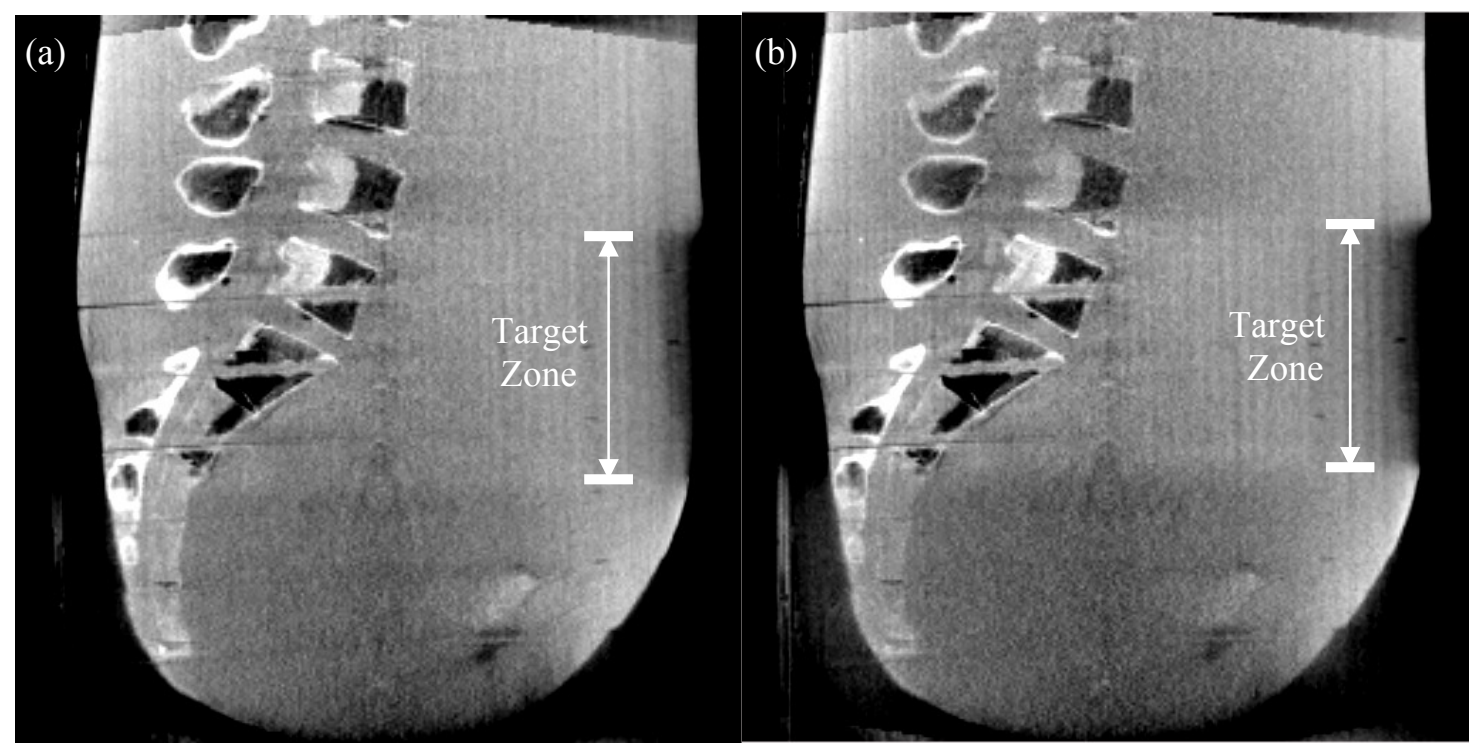

Figure 12: Sagittal $1 \mathrm{~mm}$ slices through Rando phantom CBCT zonal filter images with $10 \mathrm{~cm}$ target zone length and filter thickness (a) $12 \mathrm{~mm}$ (b) $24 \mathrm{~mm}$. 
Figure 13 shows the success rate for automatic registration of $\mathrm{CBCT}$ to conventional $\mathrm{CT}$ as a function of standard deviation of initial offset applied to the CBCT image. For initial offsets of 2 $\mathrm{mm}$ SD all images had a $100 \%$ registration success rate. With $5 \mathrm{~mm}$ SD initial offsets both the 5 $\mathrm{cm}$ and $10 \mathrm{~cm}$ lead collimated images suffered some loss in registration robustness, while the full field and zonal filter CBCT images retained $90-100 \%$ success. For initial offsets with larger SD the lead collimated CBCTs suffered a large loss of registration robustness (success rate 20-40\%), while the full field and zonal filter images retained high success rate (70-80\%).

(a) $10 \mathrm{~cm}$ target zone length

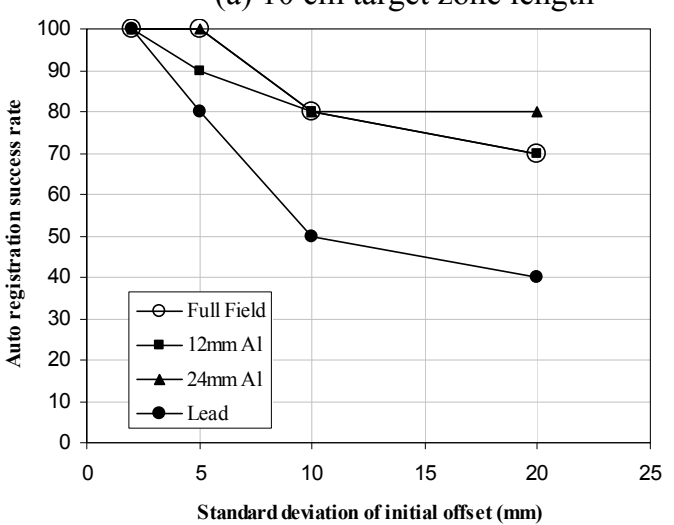

(b) $5 \mathrm{~cm}$ target zone length

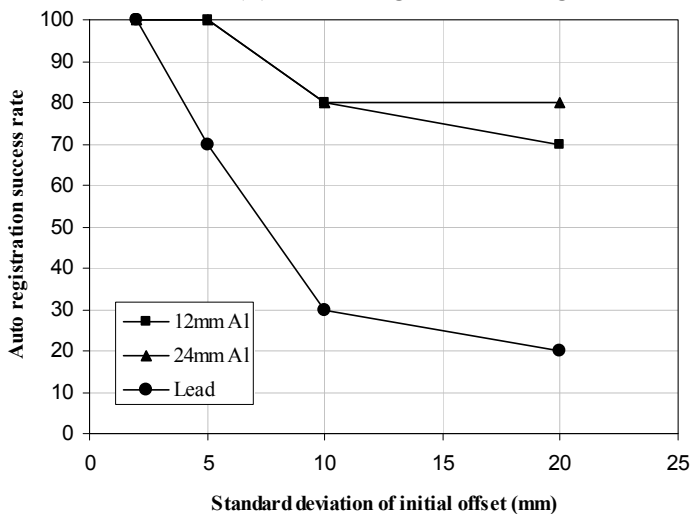

Figure 13: Success rate of automatic registration as a function of standard deviation of initial offset for (a) target zone length $10 \mathrm{~cm}$ (b) target zone length $5 \mathrm{~cm}$. The different symbols represent zonal filter thicknesses. Also shown in (a) are the results for the full $25 \mathrm{~cm}$ field of view length.

\section{Discussion}

The use of transmission diaphragms mounted at the X-ray source as a zonal filter has produced useful improvements in CNR in the tumour target zone. This has been achieved due to the reduction in scattered radiation reaching the panel from the partially collimated setup zone. The improvement in CNR from cutting scattered radiation is explained by an increase in contrast, which outweighs the increase in statistical noise due to lower overall exposure at the detector panel, as reported by Siewerdsen and Jaffray (2001). The central part of the imaging beam has not been modified and hence the change in contrast has not been contributed to by a change in energy spectrum.

There is a reduction in CNR in the setup zone, which is as expected due to the heavily attenuated primary beam. However, the high contrast spatial resolution measurements show that edge detail for high contrast objects, such as bone, has not been degraded by use of the zonal filter.

The range of robustness for automatic registration is increased with the wider-field edge detail provided by the zonal filter. High success rate is maintained using the zonal filter for initial offsets with standard deviation of $2 \mathrm{~cm}$, sufficient to encompass the majority of set-up deviations expected in pelvic radiotherapy. The robustness of automatic CBCT to planning CT image registration is of vital importance to the success of on-line image-guided set-up protocols, as manual image matching is significantly slower. Longer times between imaging and treatment increase the probability of patient or organ movement, decreasing confidence in the corrections determined. Increased treatment times would also limit the applicability of the technique in busy radiotherapy departments. 
Significant dose reduction at all points has been shown when using the zonal filter, relative to full field imaging, not only in the setup zones under the filter but also at the centre of the field regardless of location. Reduction in dose in the target zone is purely due to the cutting of scattered radiation from the setup zones of the imaging field. The large central dose reduction here, of around $50 \%$, reflects the significant contribution of scattered radiation in CBCT. The amount of scatter increases with the field size, and also with the size of the subject. This was studied by Siewerdsen, et. al. (Siewerdsen and Jaffray, 2001), who found a scatter-to-primary ratio (SPR) of radiation reaching the panel of $110 \%$ in pelvic imaging for fan angle $14^{\circ}$ and cone angle $7^{\circ}$. Elekta Synergy has fan and cone angles both $15^{\circ}$ for the full FOV, hence SPR of greater than $100 \%$ can be expected, which is consistent with the reduction in dose of around $50 \%$ from cutting the scatter contribution.

A dual exposure technique is described by Letourneau, et. al. (Letourneau et al., 2005), in which a low-dose, large-field CBCT scan is followed by a small-field, "local tomography" scan of the target area. The two scans are superimposed to give wide-field patient surface and bony anatomy information, with improved image contrast in the target area. This type of image could be achieved more quickly and at lower dose, in single scan using the zonal filter method with the transmission filter oriented parallel to the axis of rotation. Further studies are underway at our institution to investigate this possibility, and also to assess the utility of the zonal filter technique in patient CBCT scans. Also the subject of a further study is the optimization of zonal filter material and thickness, to give the maximum reduction in imaging dose, while retaining sufficient wide field detail for robust image registration of bony anatomy.

\section{Conclusion}

This work has shown that for wide field cone beam tomography the removal of scatter at source in the patient has multiple benefits. A novel zonal transmission filter is introduced to this effect. It is shown to provide improved CNR where it is most desirable, in the target zone, and significantly reduced patient dose relative to full-field $\mathrm{CBCT}$ imaging, whilst retaining the widefield bony anatomy information necessary for robust automatic image registration.

\section{Acknowledgements}

The authors would like to thank Elekta Oncology Systems and the Christie Hospital Charitable Funds for supporting the program of Image Guided Radiotherapy Research at the Christie Hospital.

\section{References}

Aird E G 2004 Second cancer risk, concomitant exposures and IRMER(2000) Br J Radiol 77 983-5

Amer A, Marchant T, Sykes J R, Czajka J, Davies J, Stratford J, McCarthy C, Henry A, McBain $\mathrm{C}$ and Moore C J 2005 Doses from cone beam CT integrated to a radiotherapy treatment machine Clin Oncol 17 S11 (Abstract)

de Crevoisier R, Tucker S L, Dong L, Mohan R, Cheung R, Cox J D and Kuban D A 2005 Increased risk of biochemical and local failure in patients with distended rectum on the planning CT for prostate cancer radiotherapy Int J Radiat Oncol Biol Phys 62965

Endo M, Tsunoo T, Nakamori N and Yoshida K 2001 Effect of scattered radiation on image noise in cone beam CT Med Phys 28 469-474

EU 1997 Council Directive 97/43/Euratom Health Protection of Individuals Against the Dangers of Ionizing Radiation in relation to Medical Exposure EU

EU 1999 European Guidelines on Quality Criteria for Computed Tomography EUR 16262 Brussels EU 
Feldkamp L A, Davis L C and Kress J W 1984 Practical cone-beam algorithm J Opt Soc Am A 1 612-619

Ghilezan M, Yan D, Liang J, Jaffray D A, Wong J W and Martinez A A 2004 Online imageguided intensity-modulated radiotherapy for prostate cancer: How much improvement can we expect? A theoretical assessment of clinical benefits and potential dose escalation by improving precision and accuracy of radiation delivery Int J Radiat Oncol Biol Phys $\mathbf{6 0}$ 1602

HMSO 2000 Ionising Radiation (Medical Exposure) Regulations SI 2000/1059 UK HMSO

IEC 1994 Evaluation and routine testing in medical imaging departments - Part 2-6: Constancy tests - X-ray equipment for computed tomography 61223-2-6 International Electrotechnical Commission Geneva

Jaffray D A, Siewerdsen J H, Wong J W and Martinez A A 2002 Flat-panel cone-beam computed tomography for image-guided radiation therapy Int J Radiat Oncol Biol Phys 53 1337-1349

Letourneau D, Wong J W, Oldham M, Gulam M, Watt L, Jaffray D A, Siewerdsen J H and Martinez A A 2005 Cone-beam-CT guided radiation therapy: technical implementation Radiother Oncol 75 279-286

Marchant T, Sykes J, Amer A, Moore C, Stratford J, McCarthy C and Henry A 2004 Measurement of set-up error using image registration of X-ray Volume Images (XVI) and Treatment Planning CT Radiother Oncol 73 S229 (Abstract)

Mori S, Endo M, Nishizawa K, Tsunoo T, Aoyama T, Fujiwara H and Murase K 2005 Enlarged longitudinal dose profiles in cone-beam CT and the need for modified dosimetry Med Phys 32 1061-9

Netsch T, Rosch P, Muiswinkel A v and Weese J 2001 Towards real-time multi-modality 3D medical image registration 8th International Conference on Computer Vision (ICCV) Vancouver, BC IEEE Press

Oldham M, Letourneau D, Watt L, Hugo G, Yan D, Lockman D, Kim L H, Chen P Y, Martinez A A and Wong J W 2005 Cone-beam-CT guided radiation therapy: A model for on-line application Radiother Oncol 75 271.E1

Roach M 2005 Commentary on increased risk of biochemical and local failure in patients with a distended rectum on the planning CT for prostate cancer radiotherapy Int J Radiat Oncol Biol Phys 62949

Rosch P, Netsch T, McNutt T, Shoenbill J and Root P 2003 Syntegra Automated Image Registration Algorithms Syntegra White Paper Eindhoven Philips Medical Systems

Siewerdsen J H and Jaffray D A 2001 Cone-beam computed tomography with a flat-panel imager: magnitude and effects of x-ray scatter Med Phys 28 220-231

Siewerdsen J H, Moseley D J, Bakhtiar B, Richard S and Jaffray D A 2004 The influence of antiscatter grids on soft-tissue detectability in cone-beam computed tomography with flatpanel detectors Med Phys 31 3506-20.

Smitsmans M H P, Wolthaus J W H, Artignan X, de Bois J, Jaffray D A, Lebesque J V and van Herk M 2004 Automatic localization of the prostate for on-line or off-line image-guided radiotherapy Int J Radiat Oncol Biol Phys $\mathbf{6 0} 623$

Sykes J R, Amer A, Czajka J and Moore C J 2005 A feasibility study for image guided radiotherapy using low dose, high speed, cone beam X-ray volumetric imaging Radiother Oncol 7745 